\title{
Effectiveness of Bacteriophages in the Era of Antibiotic Resistance
}

\author{
Ramya K, Sowmiya PV, Rani N and Sankar P* \\ Veterinary College and Research Institute, India
}

*Corresponding author: Sankar P, Assistant Professor, Department of Pharmacology and Toxicology, Veterinary College and Research Institute, Namakkal, Tamil Nadu, India, Email: drpsankarster@gmail.com

\section{Review Article \\ Volume 3 Issue 2}

Received Date: March 03, 2018

Published Date: April 24, 2018

\section{Abstract}

The prognosis of many diseases in the present circumstances is often dubious or uncertain. The reason for the presentday state is the consequences of reckless role of human mankind towards nature and irresponsible use antibiotics since when the antibiotics were discovered. The health of the human beings and livestock in the near future remains obscure If the same situation prevails. Hence, the decline in the effectiveness of antibiotics warrants the exploration of novel strategies and elements to combat the emerging antimicrobial resistance globally. Bacteriophages are one such alternate for antibiotics which can be commendably used in various fields like therapeutics, bio fermentation, food processing etc.

Keywords: Bacteriophages; Bio fermentation; Anti-bacterial

\section{Introduction}

Bacteriophages or phages are bacterial viruses that invade bacterial cells and, in the case of lytic phages, disrupt bacterial metabolism and cause the bacterium to lyse. Thousands of varieties of phage exist, each of which may infect only one type or a few types of bacteria. Like all viruses, phages are simple organisms that consist of a core of genetic material (nucleic acid) surrounded by a proteincapsid. The nucleic acid may be either DNA or RNA and may be double-stranded or single-stranded. There are three basic structural forms of phage: an icosahedral (twenty-sided) head with a tail, an icosahedral head without a tail, and a filamentous form [1].

Over the past three decades, phage research has revealed the abundance of phages in nature, the diversity of their genomes, their impact on evolution of microbial diversity, their control of infectious diseases and their influence in regulating the microbial balance in every ecosystem where this has been explored, which led to the resurgence of interest in phage research.

Phages are widely distributed in locations populated by bacterial hosts, such as soil or the intestines of animals. One of the densest natural sources for phages and other viruses is sea water, where $9 \times 10^{8}$ virions per milliliter have been found in microbial mats at the surface, and up to $70 \%$ of marine bacteria may be infected by phages.

\section{Utility of Bacteriophages}

\section{Phage Therapy}

With the recent development of antibiotic resistance within the microbial population, the need for new 
antibacterials and alternative strategies to control microbial infections is of increasing urgency [2]. One possible option is the use of bacteriophage as antimicrobial agents. Lytic phage kill bacteria via mechanisms that differ from those of antibiotics, and therefore, can be considered as antibacterials with a 'novel mode of action', a concept desired for all new antibacterial agents. The use of phages to treat bacterial infections in animals and humans is an old idea. In Eastern Europe and the former Soviet Union, phage therapy has been used successfully to treat bacterial dysentery, staphylococcal lung infectionsand surgical wound infections, among others. Phage therapy was exploited for both diarrheal disease and the treatment of traumatic infections during and after World War II. During the 1920s and 1930s, therapeutic phage applications spread rapidly in response to a desperate need for treatment of bacterial infections in Western Europe and the USA. Orally administered phage preparations were reported to effectively treat patients infected with dysentery [3]. Patients suffering from staphylococcal septicemia were also successfully treated by intravenous administration of anti-staphylococcal phages. Phages were reported to reduce the severity of staphylococcal meningitis and eliminate $S$. aureus from the cerebrospinal fluid. However, with the advent of antibiotics for the management of infections in the early 1940 s and their simultaneous widespread use, early clinical trials were abandoned in the West. Currentlyrenewed interest is perceived in bacteriophage therapy in many parts of the worlddue to the emergence of drug-resistant pathogenic bacteria and there are strong indications that phages may yet have an important role to play in the treatment of bacterial infection around the globe [4].

\section{Phage Lysins as Antimicrobials}

A number of recent studies have shown the enormous potential of the use of phage endolysins, rather than the intact phage, as potential therapeutics. Phage endolysins, or lysins, are enzymes that damage the cell wall integrity by hydrolyzing the four major bonds in its peptidoglycan component.

The majority of phage lysins studied to date are modular in structure, composed of at least two distinctly separate functional domains:

- A C-terminal cell-wall binding domain, which directs the enzyme to its target.
- An N-terminal catalytic domain whichcan comprise one or more of the following types of peptidoglycan hydrolases: endopeptidases, muramidases (lysozyme), $\mathrm{N}$-acetylmuramyl- L-alanine amidases and glucosamidases. Most of the lysinsstudied to date are amidases [5].

\section{Phage Display Technology}

Phage display technology is a particularly powerful molecular tool that has had a major impact on drug discovery, pharmacology, immunology and plant science. It is a technique by which foreign peptides, proteins or antibody fragments are expressed at the surface of phage particles. The heterologous peptide or protein is cloned into a phage or phagemid genome as a transcriptional fusion with one of the coat protein genes. These phages then become vehicles for expression that not only carry within them the nucleotide sequence encoding the expressed proteins, allowing the gene sequence to be retrieved, but also have the capacity to replicate [6].

\section{Vaccines}

A novel and exciting use of phages is the use of whole phage particles to deliver vaccines in the form of immunogenic peptides attached to modified phage coat proteins, or as delivery vehicles for DNA vaccines. Phage display is useful for the identification of immunogenic epitopes or mimotopes on displayed peptides which could, in turn, become the basis of peptide vaccines. A study carried out comparing the humoral immune response of animals immunized with a recombinant hepatitis B vaccine or with mimotopes generated by phage display demonstrated that the mimotopes could induce a response similar to that induced by the original antigen; in fact, the mimotopes induced the most reproducible and potent response. Bastien et al. investigated whether a recombinant phage displaying a known protective epitope to the human syncytial virus could protect against infectious challenge in mice. The authors reported that complete protection against the corresponding pathogen could be elicited through mucosal delivery of a filamentous phage displaying the vaccine peptide. This study supports the usefulness of phage display of defined epitopes in prophylactic vaccination. Vaccination with phagedisplaying immunogenic peptides has a number of advantages over the use of recombinant peptides, such as the stimulation of both the cellular and humoral arms of the immune system. 


\section{Open Access Journal of Veterinary Science \& Research}

\section{Detection of Pathogens}

The specific interaction of a bacteriophage and its host lends itself to using phages for the detection of bacteria, in particular, pathogenic bacteria. Unlike other detection systems such as ELISA and PCR, detection with phage is a natural system whereby the phages specifically recognize and bind to their host cells.

\section{Role of Phages in Biofilm Penetration}

Antibiotic therapy is highly effective with planktonicbacteria, such as V. cholerae and Yersinia pestis, yet is limited in treating biofilm-based bacterial infection.Phages, however, are equipped with enzymes (e.g., EPS depolymerase) on the exterior of the capsid thatdegrade the extracellular polymeric substances (EPS)and disperse bacterial biofilms, allowing the phage toaccess bacteria embedded within the EPS matrix. The phage progeny released upon completion of the lyticcycle propagate the dispersal of the biofilm through theremoval of biofilm-embedded bacteria in subsequentlayers. In order to penetrate dense biofilms, highdoses of antibiotics are typically required to observeany inhibition of bacterial growth, yet completeeradication is rare and regrowth of colonies beginsafter the end of antibiotic treatments. Althoughlow concentrations of many antibiotics are generallyconsidered non-toxic, high concentrations can result in tissue toxicity. Gabisoniya, et al. at the EliavaInstitute of Bacteriophages in Tbilisi, Georgia foundthat the application of phages on in vitro colonies of thepathogen P. aeruginosa not only prevented additionalbiofilm formation by the pathogen but also degradedexisting biofilm. Phage treatments have eliminatedbiofilms formed by $L$. monocytogenes, $P$. aeruginosa and Staphylococcus epidermidis on the surface ofmedical devices. These findings are highly relevantto the problem of persistent infections caused byimplanted medical devices such as catheters, lenses and prostheses where biofilm formation is common.

\section{Conclusion}

In summary, bacteriophages have several characteristics thatmake them potentially attractive therapeutic agents. They are highly specific and very effective in lysing targeted pathogenicbacteria, rapidly modifiable to combat the emergence ofnewly arising bacterial threats. Phages may be alternative treatment modalitiesagainst rapidly emerging, antibiotic-resistant bacteria towarrant further studies in the field of phage therapy.Bacteriophages offer complementary approaches to conventional antibiotics and other antimicrobial agents, and they can be used in various applications ranging from food safety to therapeutics.

\section{References}

1. Wittebole X, De Roock S, Opal SM (2014) A historical overview of bacteriophage therapy as an alternative to antibiotics for the treatment of bacterial pathogens. Virulence 5(1): 226-235.

2. Yoshikawa TT (2002) Antimicrobial resistance and aging: beginning of the end of the antibiotic era? Journal of the American Geriatrics Society 50: S226S229.

3. Laxminarayan R, Duse A, Wattal C, Zaidi AK, Wertheim HF, et al. (2013) Antibiotic resistance-the need for global solutions. Lancet Infectious Disease 13(12): 1057-1098.

4. Centers for Disease Control (2015) Antibiotic Resistance: The Global Threat.

5. Luepke KH, Suda KJ, Boucher H, Russo RL, Bonney MW, et al. (2017) Past, Present, and Future of Antibacterial Economics: Increasing Bacterial Resistance, Limited Antibiotic Pipeline, and Societal Implications. Pharmacotherapy 37(1): 71-84.

6. Carlton RM (1999) Phage therapy: past history and future prospects. Archivum Immunologiae et Therapiae Experimentalis 47(5): 267-274. 